\title{
Analysis of Static Magnetic Field of Electric - Machine Converter
}

\author{
Changke Tan ${ }^{1, a^{*}}$, Chonglou Tong ${ }^{2, b}$, Fei Zhou ${ }^{3, c}$ and Yuankun $\mathrm{Xu}^{4, \mathrm{~d}}$ \\ College of Mechanical Engineering, Xijing University, Xi'an, China \\ a995965262@qq.com,b1205802724@qq.com, ${ }^{\mathrm{c}} 743040357$ @qq.com, ${ }^{\mathrm{d}} 812149187$ @qq.com,
}

Keywords: Electro-hydraulic Proportional Valve; Electric - Machine Converter; Static Magnetic Field; Permanent Magnet Material

\begin{abstract}
Electricity-machinery converter is the input unit of electro-hydraulic proportional valve and mechanical and electrical switching device, and the input electrical signal is proportionally converted into mechanical quantity by electricity-machinery converter. The moving coil electromechanical converter is used as the main research object in this paper. The static magnetic fields of electric machine converter are analyzed that includes four aspects. These aspects include the structure of thrust coil framework, the permanent magnet material, single magnetization technique of permanent magnet, a number of permanent magnet magnetization array structures.
\end{abstract}

\section{Introduction of Electric - Machine Converter}

With the rapid development of hydraulic transmission technology, electro-hydraulic proportional valve as a representative of the electric - machine conversion component which has been widely used in mechanical engineering. Electric - machine converter as input unit of electro-hydraulic proportional valve and mechanical and electrical switching device, the input signal (voltage, current) is continuous proportionally converted into mechanical quantity (force, torque or displacement).

The type of Electric - Machine Converter. According to the structure of movable parts, electric-machine converter is made up of moving iron type and moving coil type [1].

(1) The moving iron type electric-mechanical converter is made up of armature coils, shell, etc. Input signal is processed by amplifier, loaded into the control winding and armature magnetic field generated in a current-carrying coil in the electromagnetic force and displacement, so as to drive the valve core movement. The electric-machine converter of moving iron type possesses the advantages of inductive load big, the driving force [2]. But because of the moving parts of inertia is big, so it can only be as a control switch system or actuators in low-frequency and low control precision.

(2) The electromechanical converter of moving coil is widely concerned with its high linearity, hysteresis and good response characteristic. The electromechanical converter of moving coil can produce 2.5 times of the electromagnetic force in same electromagnetic valve. But the traditional structure of the coil, eddy current is generated in magnetic materials, and it can reduce the electromagnetic wire coil in the process of movement.

The Analysis of Static Magnetic Field of Electric - Machine Converter. Improve the performance of moving coil of electromechanical converter, such as frequency response, linearity and load ability. The performances help to improve the overall characteristics of the electro hydraulic proportional valve system. Static magnetic field characteristics of the converter so the moving coil electromechanical converter is the research object in static magnetic field of electric - machine converter. The static magnetic field analysis of moving coil electromechanical converter mainly includes: structure of thrust coil framework, permanent magnet materials, single magnetization technique of permanent magnet and a number of permanent magnet magnetization array structure. 


\section{Thrust Coil Skeleton Structure}

Thrust coil skeleton is a moving part of electric-mechanical converter, the coil skeleton is connected to the valve core, and the control coil is installed on the thrust coil skeleton [3]. Electricity generated by permanent magnet coil constant magnetic field by the effect of electromagnetic force, thus coil skeleton is driver to movement, the valve core is move, and the valve port is open. The characteristics of thrust coil framework directly affects the performance of the whole electric mechanical converter, to improve the structure of thrust coil framework, to improve its quick response performance, the main measures is taken from the following two aspects:

(1) Reduce the quality of the moving parts, reduce the inertia, and improve its athletic performance.

(2) Reduce the air resistance of thrust coil framework, in order to reduce the electricity a mechanical driving force of the converter.

In terms of reducing the weight of moving parts, it is mainly to control coil and thrust coil skeleton material selection. Among them, the coil adopts the enameled wire, skeleton adopts ANB79 aluminum alloy, and the material has high strength and high quality characteristics, so it is suitable for precision components.

In terms of reducing air resistance, the current plan work out several holes on the thrust coil skeleton. In this way, not only the quality of the moving parts is reduced, but also when the thrust coil skeleton high-speed mobile, the air flow can rise to balance the action.

\section{Permanent Magnet Material}

The permanent magnet of electric - machine converter is provided for magnetic circuit, and the magnetic field intensity and magnetic induction intensity are provided which is change, and it is in accordance with the permanent magnet demagnetization curve in the process of running of electric machine converter [4]. Conventional moving coil electromechanical converter in one use of permanent magnetic material, aluminum nickel is usually used. Its biggest disadvantage is that the double line back do not overlap with the demagnetization curve, the calculation is difficulty.

When the moving coil electromechanical converter make, iron boron $(\mathrm{NdFeB})$ permanent magnet materials permanent magnets is used and the material characteristic is that the demagnetization curve is a straight line. Therefore, the magnetic of mechanical electrical converter can remain stable in operation. Manufacturing process of $\mathrm{NdFeB}$ include sintering, iron boron magnet and hot-pressing sintering method. Consolidation is formed isotropic magnet after sintering. Hot-pressing sintering method heat fused into crystal magnet under high temperature and high pressure, its performance is better, and the application is more extensive.

\section{Magnetization Technique of Permanent Magnet}

Magnetization technique of coil electromechanical converter is analysed, including different permanent magnet magnetization direction of current-carrying coil and permanent magnet inter-atomic forces.

High performance permanent magnet is made into cylindrical commonly, because the cylindrical model casting and stamping is easy, and the grain orientation of the electromagnetic coil is also very convenient. The magnetization principle of permanent magnet is shown in Fig. 1. Permanent magnet is placed between the poles of magnetizing apparatus, and the current in the electric coil is increased from scratch [5]. The magnetic flux is by permanent magnets, and the ultimate magnet magnetization process is completed.

At present, a mechanical moving coil electromechanical converter uses the permanent magnet the magnetization technology, and generally uses the axial magnetization, but this is not the best choice. Change the direction of magnetization [6], polarized magnetic flux and the coil of the external magnetic field direction is almost the same, B between the coil and magnet force will be improved. 


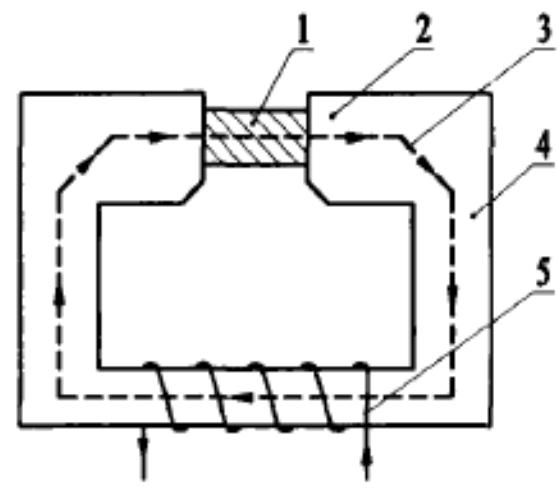

1 - Permanent Magnet; 2 -Pole; 3 Magnetic Flux; 4 Charger Magnetizer; 5 - Electric Coil

Fig. 1 Principle Diagram of Permanent Magnet Magnetization

The permanent magnet is placed in the uniform magnetic field when magnetic induction intensity $\mathrm{B}$ is constant, as shown in Fig. 2. The permanent magnet can be regarded as the combination of magnetic dipole, each magnetic dipole is independent of the magnetic field of magnetic moment $\mathrm{m}$, and the sum total of all on magnetic force is the force of permanent magnet [7].

In the gradient field, the force $\mathrm{F}$ of permanent magnet poles:

$$
F=(m \cdot \nabla) B=m_{x} \frac{\partial B}{\partial x}+m_{y} \frac{\partial B}{\partial y}+m_{z} \frac{\partial B}{\partial z}
$$

In equation (1): $\mathrm{m}_{\mathrm{x}}$ - magnetic moment $\mathrm{m}$ on the $\mathrm{x}$ axis component;

$\mathrm{m}_{\mathrm{y}}$ - magnetic moment $\mathrm{m}$ on the $\mathrm{y}$ axis component;

$\mathrm{m}_{\mathrm{z}}$ - magnetic moment $\mathrm{m}$ on the $\mathrm{z}$ axis component;

B - Induction strength of external magnetic field magnetic.

When the magnetic fields caused by magnetic current have been ignored, Equation (1) can be simplified as:

$$
F=\operatorname{grad}(m \cdot B)
$$

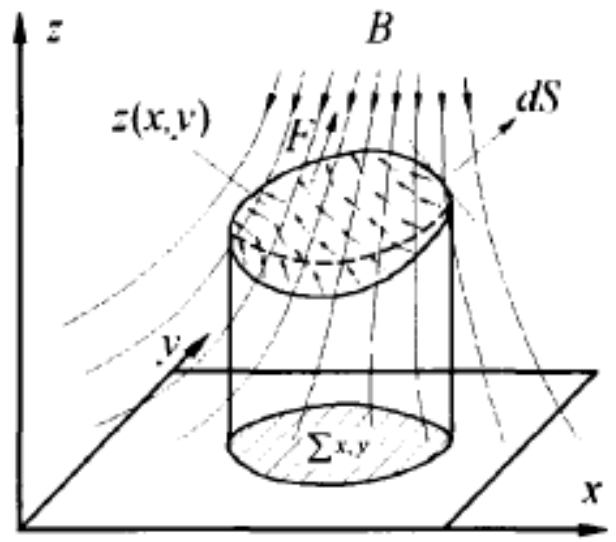

Fig. 2 Permanent Magnet Magnetization Directions in Gradient Magnetic Field

The relationship is shown in Fig. 3 between the Magnetic moment m, magnetic induction intensity $\mathrm{B}$, magnetic field gradient grad B, and the force $\mathrm{F}$ by the equation (2). As can be seen from Fig. 3, when the direction of magnetic moment $\mathrm{m}$ and magnetic field lines are the same, the force is biggest. Therefore, when all the direction of magnetic dipole magnetic moment and the direction of magnetic field lines are the same, their resultant force is also the biggest [8]. 


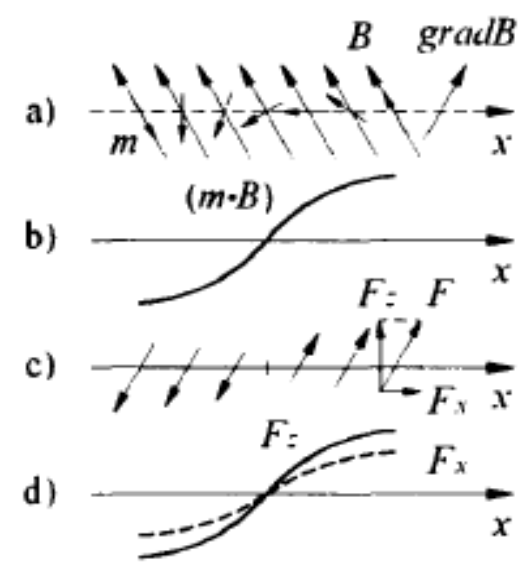

Fig. 3 Diagram of Relationship between B, F and gradB

\section{Array Structure of Permanent Magnet}

Combination of conventional permanent magnet array used one-way, the radial or tangential array structure, its magnetic field lines are shown in Fig. 3 (a) Fig. 3(c). Halbach array is a new type of permanent magnet magnetization arrangement, and it broke the traditional way of radial and tangential magnetic steel array. The radial and tangential array is together [9]. Side of the magnetic field of permanent magnet is enhanced, while the other side of the magnetic field is abating, single magnetic field intensity was increased effectively [10].

The permanent magnet moving coil of electromechanical converter is no iron core, magnetic flux leakage is small, eddy current loss is smaller, and the coil air gap magnetic induction intensity is larger. Halbach array magnetization distribution of magnetic field lines is shown in Fig. 3 (d).

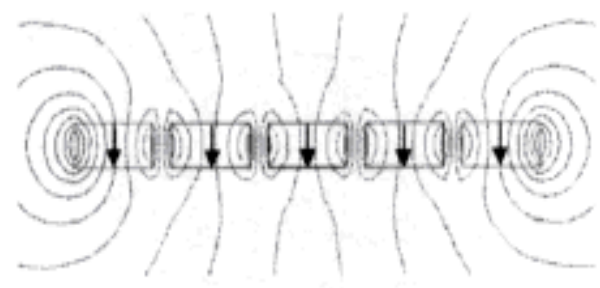

(a) unidirectional array

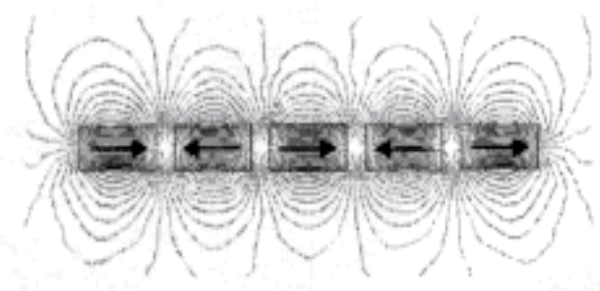

(c) tangential array

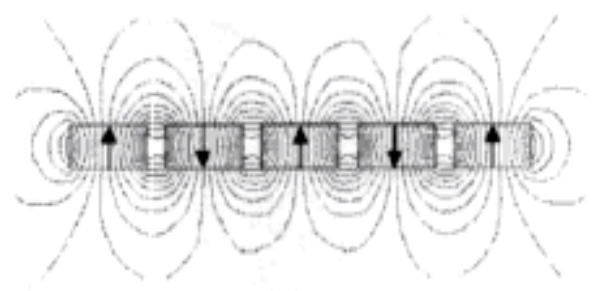

(b) radial array

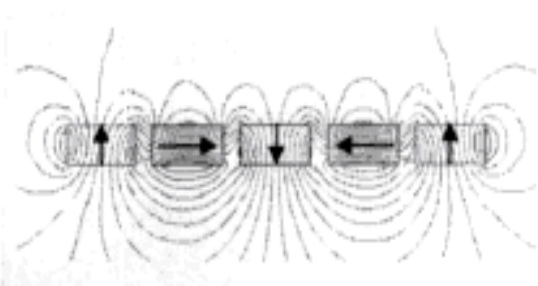

(d) Halbach array

Fig. 3 Distribution of Magnetic Force's Lines of Different Magnetization Array 


\section{Conclusions}

Based on the introduction to a mechanical electrical converter in this article, the static characteristic analysis method of mechanical electrical converter is put forward. The moving coil electromechanical converter is the main research object, and the static magnetic field of electric machine converter is analyzed from the structure of thrust coil framework, the permanent magnet material, single magnetization technique of permanent magnet, a number of permanent magnet magnetization array structures of the four directions. The static characteristic analysis of electromechanical converter is of important significance for mechanical moving coil performance index has increased and the electro-hydraulic proportional valve develops.

\section{References}

[1] M.L.Yi, S.P.Cao, Y.S.Liu. Electro-hydraulic Control Technology. Huazhong University of Science and Technology Publishing House, Beijing, 2010.

[2] J.Q.Guan, J.Q.Wen. Mechanical and Electrical Hydraulic Control Technology. Tongji University Press, Shanghai, 2010.

[3] G.Zhang, L.Y.Yu, H.W.Wu, J.Z. Electro-hydraulic Proportional Valve Research Review. Journal of Fluid Transmission and Control. 6 (2008) 1-4.

[4] Arvi Kruusing. Optimizing Magnetization Orientation of Permanent Magnets for Maximal Gradient Force. Journal of Magnetism and Magnetic Materials. 234(2001) 550-551.

[5] E.Z.Yang, X.Y li, Q.J.Sun. Proportion/Servo Valve to Make Machine More Intelligent. Modern Manufacturing. 14 (2006) 26 - 30.

[6] Tan Songtao Yu Lanying, wang etc. Ultra-high Speed Proportional Electromagnet Static Study. Journal of Hydraulic and Pneumatic. 7(2007) 49-51.

[7] J. Topfer, V Christoph. Multi-pole Magnetization of NdFeB Sintered Magnets and Thick Films for Magnetic Micro Actuators. Sensors and Actuators. 113(2011)257-263.

[8] W.T.Wu. Electro-hydraulic Proportional Control Technology Review. Power Rechnology Application. 8 (2013). 405.

[9] X.Z, K.P.Hu. The Development and Application of Electro-hydraulic Proportional Control Technology field. Association for Science and Technology BBS. (2011) 10.

[10] W.Yang. The Application of Ergonomics in the Vibratory Roller Design [J]. (2011)124-125. 